\title{
Conditional ablation of HDAC3 in islet beta cells results in glucose intolerance and enhanced susceptibility to STZ-induced diabetes
}

\author{
Wen-Bin Chen ${ }^{1,2,3}$, Ling Gao ${ }^{2}$, Jie Wang ${ }^{1,3,4}$, Yan-Gang Wang4, Zheng Dong ${ }^{5}$, Jiajun \\ Zhao' ${ }^{6}$, Qing-Sheng $\mathrm{Mi}^{1,3,7}$ and Li Zhou ${ }^{1,3,7}$ \\ ${ }^{1}$ Henry Ford Immunology Program, Henry Ford Health System, Detroit, MI, USA \\ ${ }^{2}$ Central Laboratory, Shandong Provincial Hospital Affiliated to Shandong University, Jinan, China \\ ${ }^{3}$ Department of Dermatology, Henry Ford Health System, Detroit, MI, USA \\ ${ }^{4}$ Department of Endocrinology, Affiliated Hospital of Qingdao University, Qingdao, China \\ ${ }^{5}$ Department of Cellular Biology and Anatomy, Augusta University, GA, USA \\ ${ }^{6}$ Department of Endocrinology, Shandong Provincial Hospital affiliated to Shandong University, Jinan, China \\ 7 Department of Internal Medicine, Henry Ford Health System, Detroit, MI, USA \\ Correspondence to: Qing-Sheng Mi, email: qmil@hfhs.org
}

Jiajun Zhao, email: jjzhao@medmail.com.cn

Li Zhou, email: Izhoul@hfhs.org

Keywords: HDAC3; knockout; autoimmune diabetes; glucose tolerance; insulin; Pathology Section Received: April 15, $2016 \quad$ Accepted: August 10, $2016 \quad$ Published: August 15, 2016

\section{ABSTRACT}

Histone deacetylases (HDACs) are enzymes that regulate gene expression by modifying chromatin structure through removal of acetyl groups from target histones or non-histone proteins. Previous in vitro studies suggest that HDACs may be novel pharmacological targets in immune-mediated islet $\beta$-cell destruction. However, the role of specific HDAC in islet $\beta$-cell development and function remain unclear. Here, we generated a conditional islet $\beta$-cells specific HDAC3 deletion mouse model to determine the consequences of HDAC3 depletion on islet $\beta$-cell differentiation, maintenance and function. Islet morphology, insulin secretion, glucose tolerance, and multiple lowdose streptozotocin (STZ)-induced diabetes incidence were evaluated and compared between HDAC3 knockout and wild type littermate controls. Mice with $\beta$-cell-specific HDAC 3 deletion displayed decreased pancreatic insulin content, disrupted glucosestimulated insulin secretion, with intermittent spontaneous diabetes and dramatically enhanced susceptibility to STZ-induced diabetes. Furthermore, islet $\beta$-cell line, MIN6 cells with siRNA-mediated HDAC3 silence, showed decreased insulin gene transcription, which was mediated, at least partially, through the upregulation of suppressors of cytokine signaling 3 (SOCS3). These results indicate the critical role of HDAC3 in normal $\beta$-cell differentiation, maintenance and function.

\section{INTRODUCTION}

Diabetes mellitus is a group of multi-factorial metabolic diseases characterized by absolute or relative deficiencies in insulin production. Molecular mechanisms affecting pancreatic $\beta$-cell differentiation, proliferation, survival, and function are fundamental for understanding the pathogenesis of both type 1 and type 2 diabetes. Preserving a functional $\beta$-cell mass and promoting the $\beta$-cell differentiation, proliferation and function are therefore the primary targets of novel treatments aimed at curing and preventing diabetes mellitus.

Histone deacetylases (HDACs) are enzymatic components of large multi-protein complexes that remove acetyl groups from lysine side chains of histones and other proteins. They contribute to epigenetic programming and regulation of gene expression during development and throughout life. Gene transcription is modulated by acetylation and deacetylation of histones. Acetylation of histones generally contributes to the formation of a 
transcriptionally competent environment by relaxing the chromatin structure, allowing transcription factors to access the target DNA. In contrast, histone deacetylation compacts chromatin and leads to transcriptional repression [1] Recent studies also suggested that HDACs regulate the activity of a wide range of non-histone proteins [2], thus protein acetylation has a big impact in posttranslational regulation of proteins.

Mammalian HDACs are classified into four groups on the basis of their structure and function. HDAC3 is part of class I, which also includes HDAC1, 2, and 8 [3]. Although class I HDACs are broadly expressed and structurally related, they have distinct developmental roles $[4,5]$. All HDACs were found expressed in rat $\beta$-cell line, INS-1 cells, and rat islets, implying their potential roles in islet $\beta$-cell development and function [6]. Different HDACs participate in specific multiprotein repressive transcriptional complexes, which could potentially determine, at least in part, their unique functions.

HDAC3 is a component of the nuclear receptor co-repressor complexes that contain N-CoR and SMRT [7]. Using cell specific HDAC3 deletion mouse models, previous studies have revealed the critical roles of HDAC3 in cardiac functional maintenance and myocardial energy metabolism [8], liver metabolic homeostasis [9, 10], and in bone formation and bone marrow adipocyte differentiation [11]. Previous studies suggest that HDACs may be novel pharmacological targets in immune-mediated $\beta$-cell destruction leading to type-1 diabetes. HDAC inhibitors (HDACi) reduce cytokine induced $\beta$-cell toxicity and restore insulin secretion both in vitro and in vivo $[6,12-$ 14], in which class I HDACs, especially HDAC1 and HDAC3 may play essential role [13, 15]. However, the specific role of HDAC3 in islet $\beta$-cell development, differentiation, maintenance and function remain unclear. Here, we made a conditional islet $\beta$-cells specific HDAC3 deletion mouse model to determine the consequences of HDAC3 depletion on islet $\beta$-cell differentiation, maintenance and function.

\section{RESULTS}

\section{Deletion of HDAC3 in islet $\beta$-cells in mice}

$\beta$-cell specific HDAC3 knockout mice (RIP-Cre ${ }^{+}$. HDAC $3^{\mathrm{fl} / \mathrm{fl}}$ ) were generated by mating mice expressing the Cre recombinase gene under the control of rat insulin 2 gene promoter (RIP-Cre ${ }^{+}$) with $\mathrm{HDAC}^{\text {flox/flox }}$ mice [10] to obtain RIP-Cre ${ }^{+}$HDAC $3^{\mathrm{f} / /+}$ mice, which were then crossed with $\mathrm{HDAC}^{\mathrm{f} / \mathrm{fl}}$ to get $\mathrm{RIP}-\mathrm{Cre}^{+}$.HDAC3 ${ }^{\mathrm{f} / \mathrm{fl}}$ knockout (KO) mice (Figure 1A). Littermates carrying HDAC3 $3^{\mathrm{f} /}$ fl, without cre expression (RIP-Cre- HDAC3 ${ }^{\mathrm{f} / \mathrm{fl}}$ ) were used as wild type (WT) controls. Since RIP-Cre transgenic mice have been suggested to show glucose intolerance, heterozygous RIP-Cre ${ }^{+}$HDAC $3^{\mathrm{f} /+}$ (HET) mice were also used as control for glucose tolerance experiment. HDAC $3^{\text {flox/flox }}$ and RIP-Cre alleles were identified by PCR with related primers (Table S1) (Figure 1B). To confirm HDAC3 deletion in islet $\beta$-cells from RIP-Cre ${ }^{+} \mathrm{HDAC}^{\mathrm{f} / \mathrm{fl}}$ (HDAC3 KO) mice, dispersed islet cells from these mice and WT control littermates (RIP-Cre HDAC $^{\mathrm{f} / \mathrm{fl}}$ ) were co-stained for HDAC3 and insulin. As shown in Figure $1 \mathrm{C}$, insulin and HDAC3 co-expression was observed in dispersed WT $\beta$-cells, while none of the insulin expressing $\beta$-cells express HDAC3 in dispersed $\beta$-cells from HDAC3 $\mathrm{KO}$ mice, indicating the efficient $\beta$-cells specific HDAC3 deletion in these mice (Figure 1C).

\section{Spontaneous glucose metabolism features of RIP- $\mathrm{Cre}^{+}$HDAC3 $^{\mathrm{A} / \mathrm{fl}}$ mice}

Throughout the monitoring time ( 5 to 25 weeks of age), HDAC3 KO mice showed a moderately decreased body weight compared with the WT control littermates (Figure 2C). Meanwhile, HDAC3 KO mice displayed steady higher blood glucose levels, , starting at 6 weeks old, especially before 10 weeks old (Figure 2A and 2B). Fasting blood glucose levels were measured and compared between HDAC3 KO and WT control mice. All WT mice showed normal fasting glucose levels, while 2 out of 12 HDAC3 KO mice showed elevated fasting glucose levels. Nevertheless, the overall fasting blood glucose level in HDAC3 $\mathrm{KO}$ group showed no significant difference comparing to that of WT control group (Figure 2 D).

\section{Glucose intolerance and impaired glucose- stimulated insulin secretion}

Glucose tolerance tests (GTT) was performed to assess the body's response to glucose, including insulin secretion capacity. Male mice were i.p. injected with glucose of 1 or $2 \mathrm{~g} / \mathrm{kg}$ of body weight, and impaired glucose clearance was observed in RIP-Cre ${ }^{+} \mathrm{HDAC}^{\mathrm{f} /}$ ${ }^{\mathrm{fl}}$ mice at both doses compared to WT controls (Figure $2 \mathrm{E}$ and $2 \mathrm{~F}$ ). Glucose tolerance in RIP-Cre ${ }^{+} \mathrm{HDAC}^{\mathrm{fl} /+}$ mice was indistinguishable from RIP-Cre-HDAC $3^{\text {flox/flox }}$, indicating that glucose homeostasis was not affected by HDAC3 heterozygosity or RIP-Cre expression in $\beta$-cells. Insulin secretion following the glucose administration was measured. At 5 and 30 min post glucose injection, HDAC3 KO mice showed impaired insulin secretion compared to WT controls which is consistent with increased glucose levels in the same animals (Figure 2F). 
Increased susceptibility to multiple low-dose streptozotocin (MLD-STZ)-induced diabetes

To study the sensitivity of $\beta$-cell to toxicity and inflammation induced death, we set out to induce diabetes by MLD-STZ injections to animals. Blood glucose levels

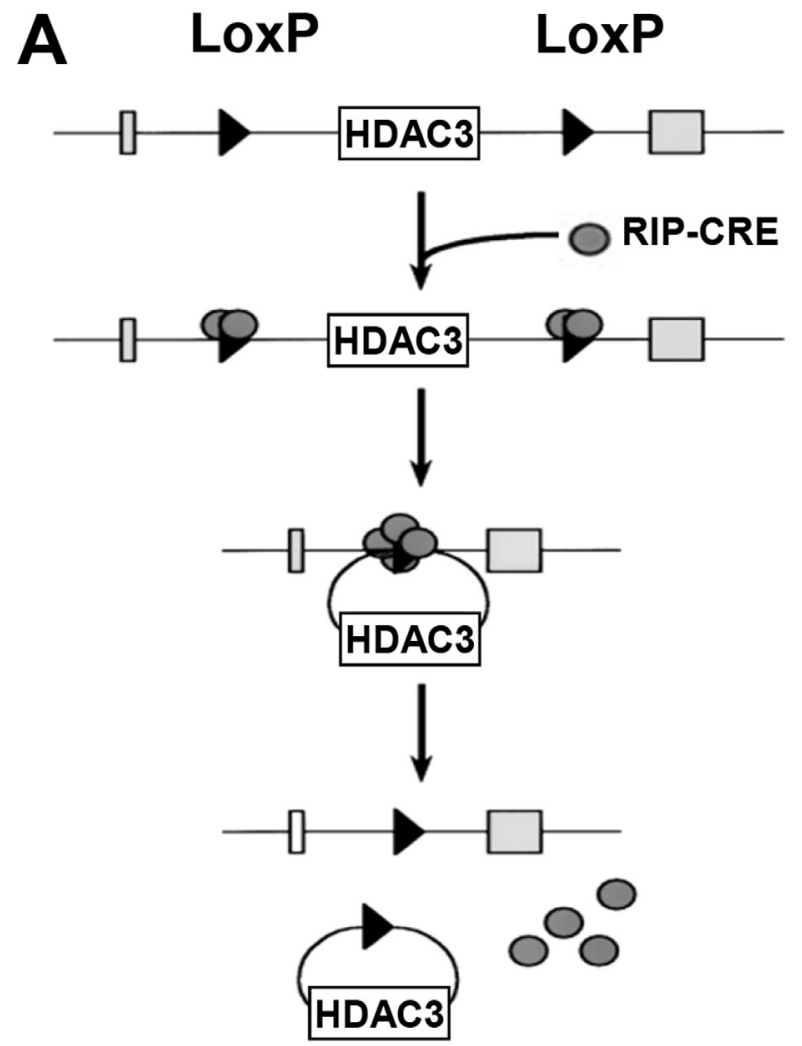

of HDAC3 KO mice showed dramatic increase compared to WT controls from day 8 till the end of the experiment (Figure 3A). When separating male and female mice into individual groups, the increased blood glucose levels in HDAC3 KO mice remained in both groups (Figure 3C and $3 \mathrm{E}$ ). Consistent with different blood glucose levels,<smiles>[BH3-]</smiles>
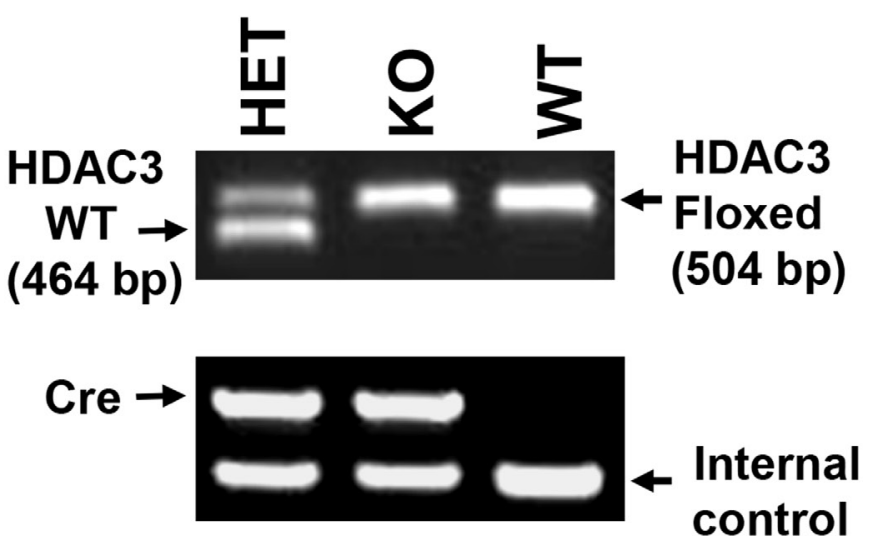
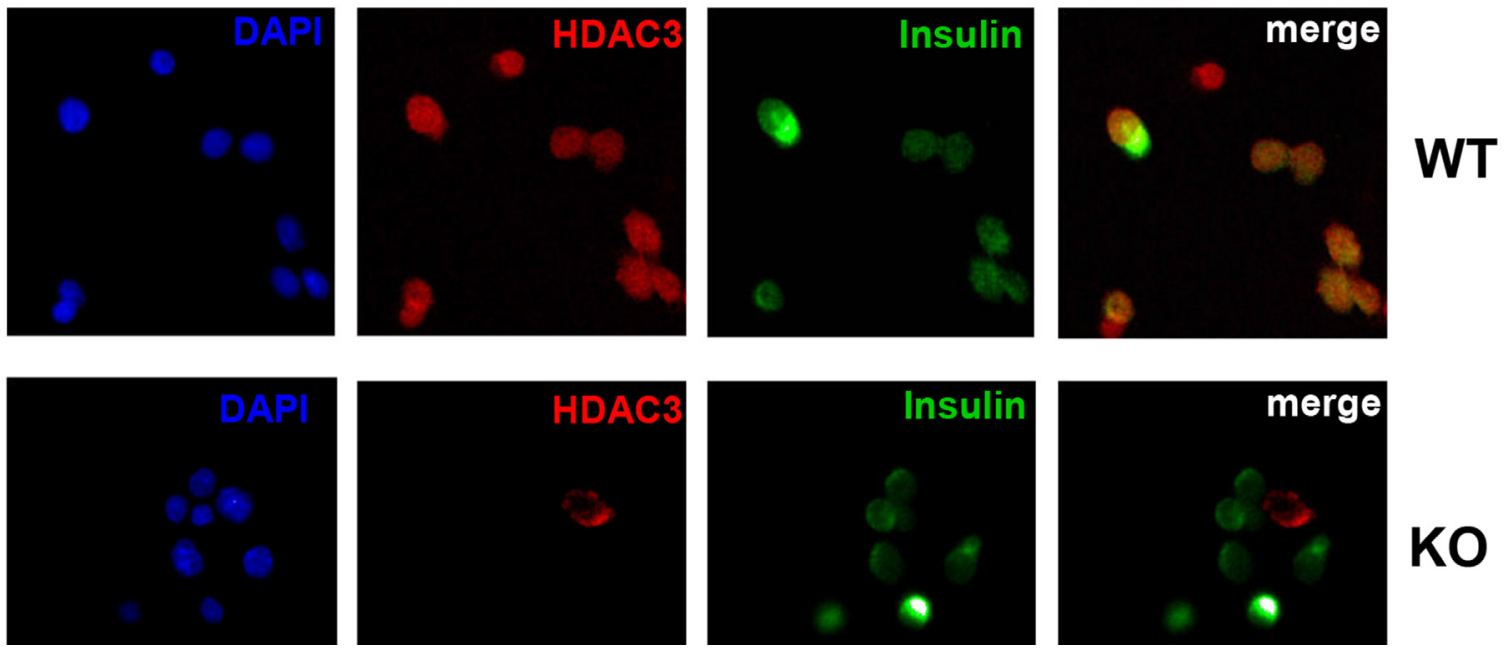

KO

Figure 1: Generation of $\boldsymbol{\beta}$-cell-specific HDAC3 knockout mice. A. The floxed and null alleles of HDAC3 as a result of recombination between LoxP sites. B. PCR results of genotyping from tail biopsies of RIP-Cre- HDAC3 ${ }^{\mathrm{ft} / \mathrm{fl}}$ (WT), RIP-Cre ${ }^{+} \mathrm{HDAC}^{\mathrm{fl} /+}$ (HET) and RIP-Cre $\mathrm{HDAC}^{\mathrm{H} / \mathrm{fl}}(\mathrm{KO})$ mice. C. Dispersed islet cells from WT and KO mice were immunostained for HDAC3 (red) and insulin (green). Yellow (merged) indicates coexpression of HDAC3 and insulin. (Scale bar, $20 \mu \mathrm{m}$ ). 

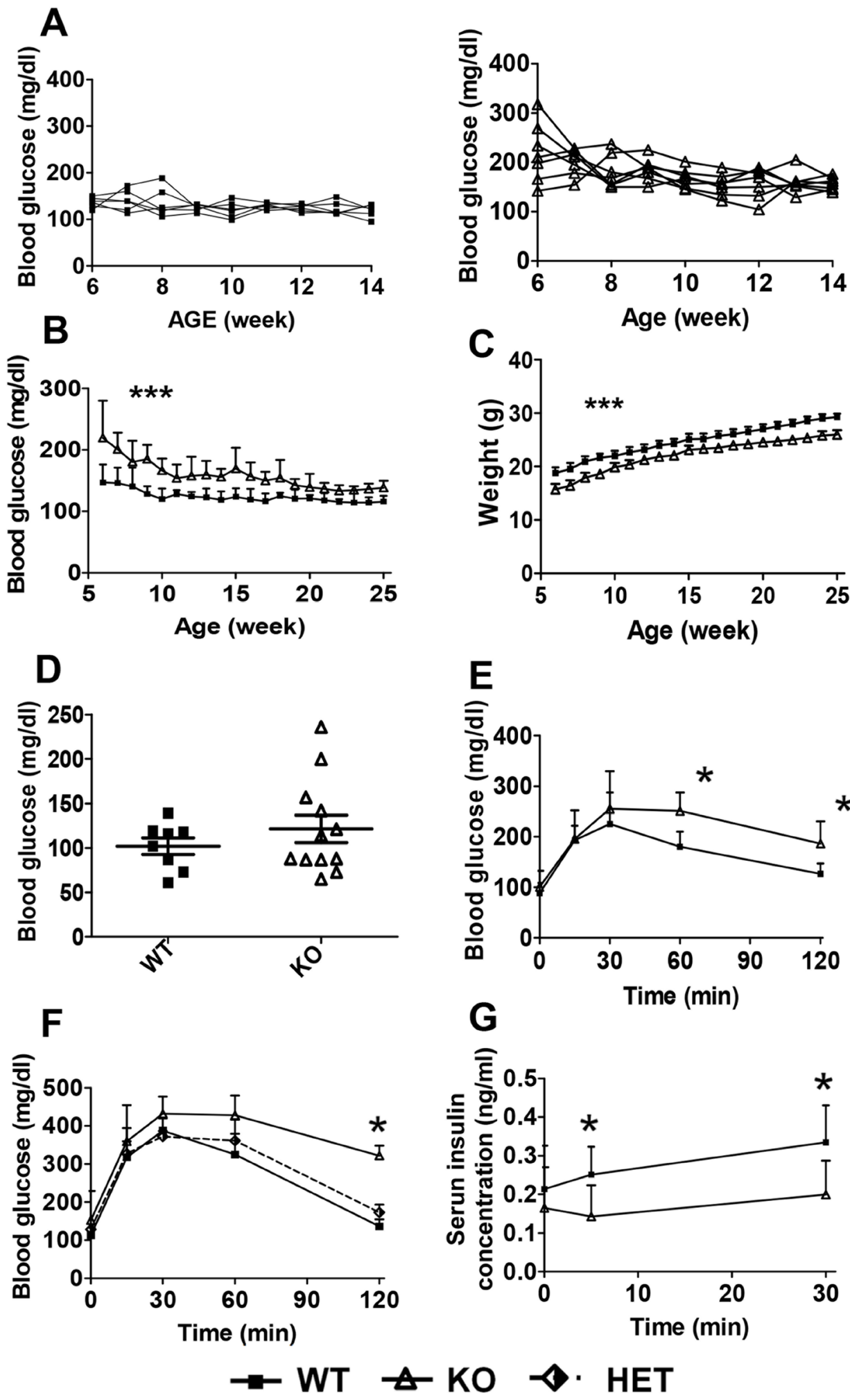

Figure 2: $\beta$-cell-specific deletion of HDAC3 causes higher blood glucose level, glucose intolerance and impaired insulin secretion in mice. A. Random blood glucose levels were monitored in WT (left) and KO (right) mice, each line represents an individual mouse. $n=6-7$ male mice/group. B. The summary of non-fasting blood glucose levels and C. body weight monitored from 6- until 25-week of age. $n=6-7$ male mice per group. The overall difference between KO and WT group was analyzed by ANOVA. D. Fasting blood glucose level was measured in 8- to 12-week-old KO and WT male mice. $n=8-12$ male mice/group. Glucose tolerance test, $1 \mathrm{~g} / \mathrm{kg}$ body weight E. or $2 \mathrm{~g} / \mathrm{kg}$ body weight $\mathbf{F}$., was performed on 8- to 10 -week-old $\mathrm{KO}$ and WT mice. $n=4-6$ mice/group. G. Plasma insulin levels during glucose tolerance test at the dose of $2 \mathrm{~g} / \mathrm{kg}$ body weight. $n=5-6$ male mice/group. Data are presented as means $\pm \mathrm{SD}$. $\left({ }^{*} p<0.05, * * * p<0.001\right)$. 
A

B
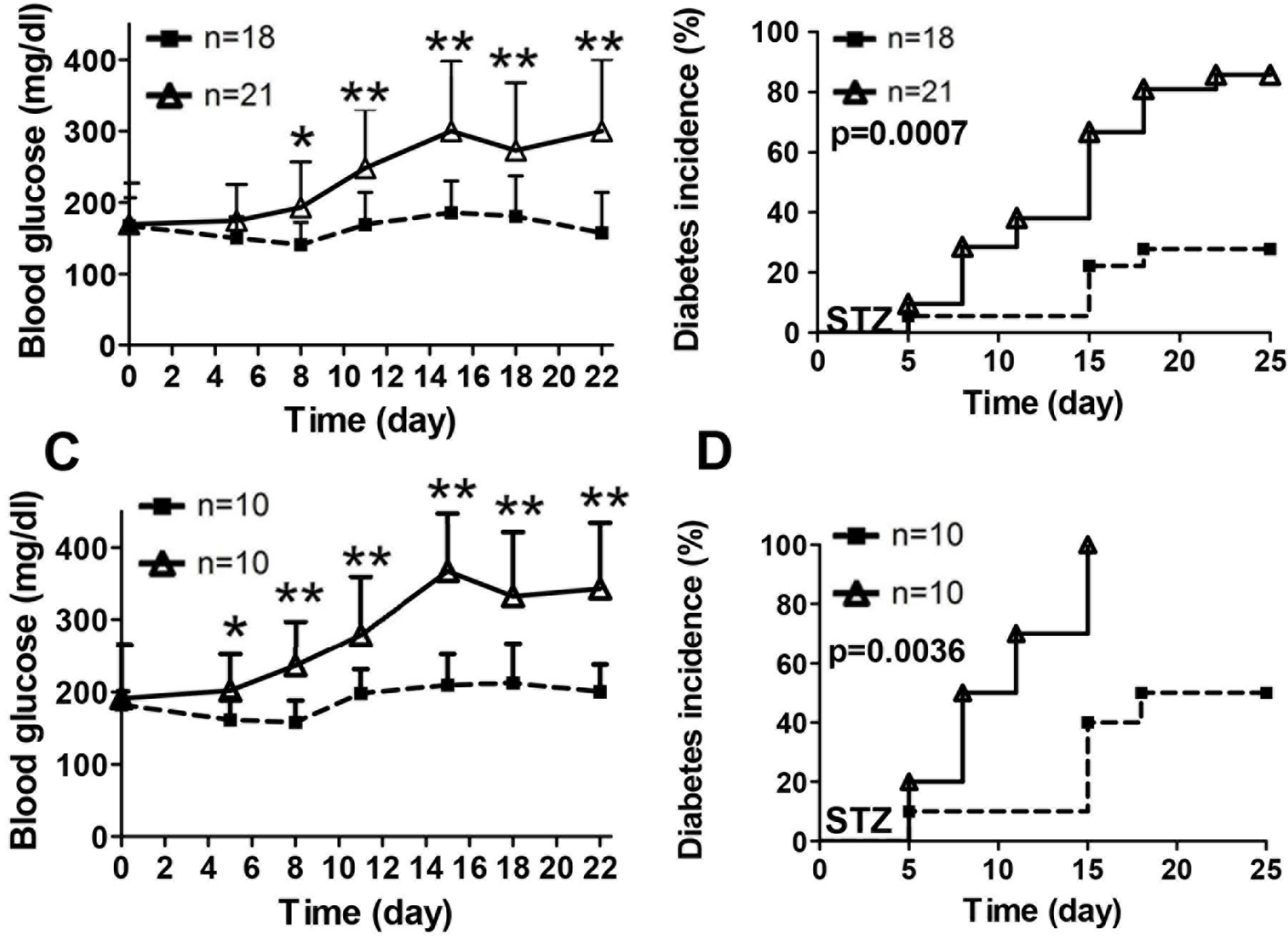

D
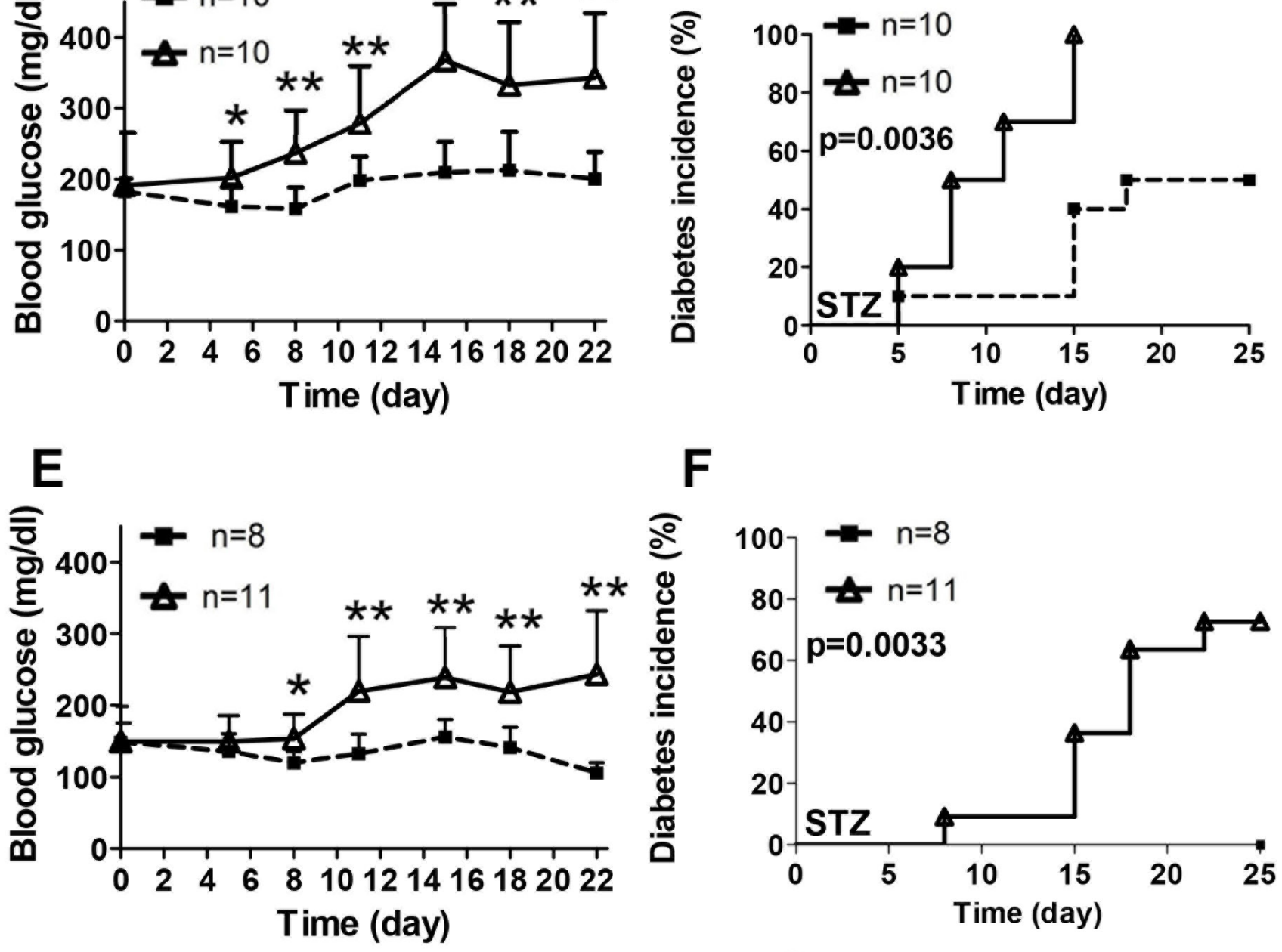

WT

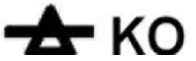

Figure 3: RIP-Cre ${ }^{+}$.HDAC3 ${ }^{\text {f/fl }}$ mice are more susceptible to multiple low-dose STZ-induced (MLD-STZ) diabetes. Blood glucose levels throughout the MLD-STZ experiment were monitored and compared between KO and WT for all mice A., male mice C. or female mice E., respectively. Diabetes incidence was monitored and compared between KO and WT mice in all mice B., male mice D. or female mice $\mathbf{F}$, respectively. Data are presented as means $\pm \mathrm{SD}$. $\left({ }^{*} p<0.05\right.$, $\left.{ }^{* *} p<0.01\right)$. 
$60 \%$ of the HDAC3 KO mice, while only $20 \%$ from WT controls developed diabetes by day 15 . By day 25 , more than $80 \%$ of HDAC3 KO mice, while only about $27 \%$ from WT controls developed diabetes (Figure 4B). Both male and female $\mathrm{KO}$ mice showed significant increased diabetes incidence compared to WT controls, respectively (Figure 3D and 3F).

\section{Decreased $\beta$-Cells and insulin content}

Relative lower body weight, hyperglycemia, glucose intolerance, and dramatically increased sensitivity to STZ treatment in HDAC3 KO mice strongly suggest a developmental and/or functional defect in pancreatic islet $\beta$-cells. Histological analysis of pancreatic sections revealed no obvious malformation of islet structure, but
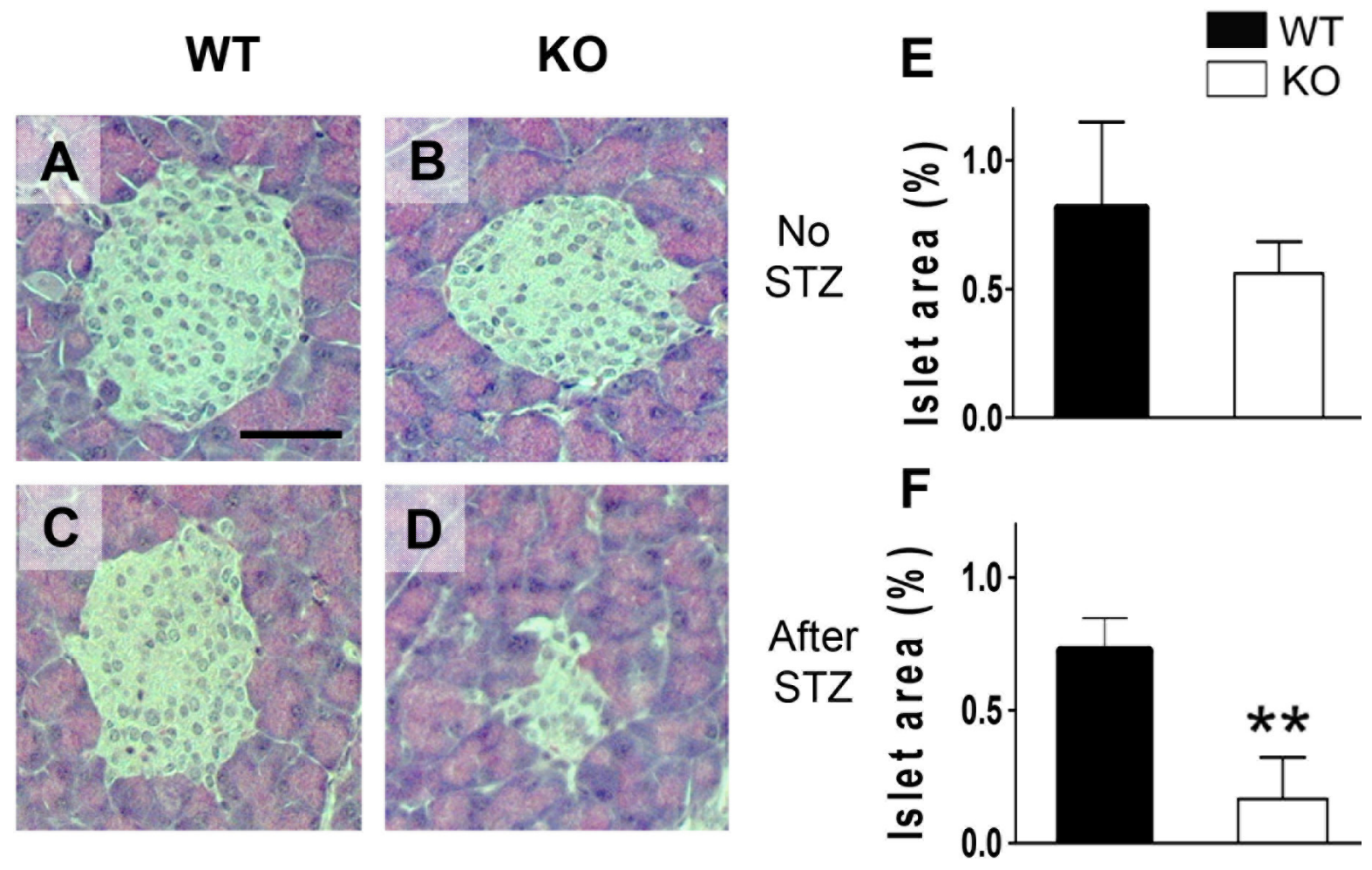

G

H
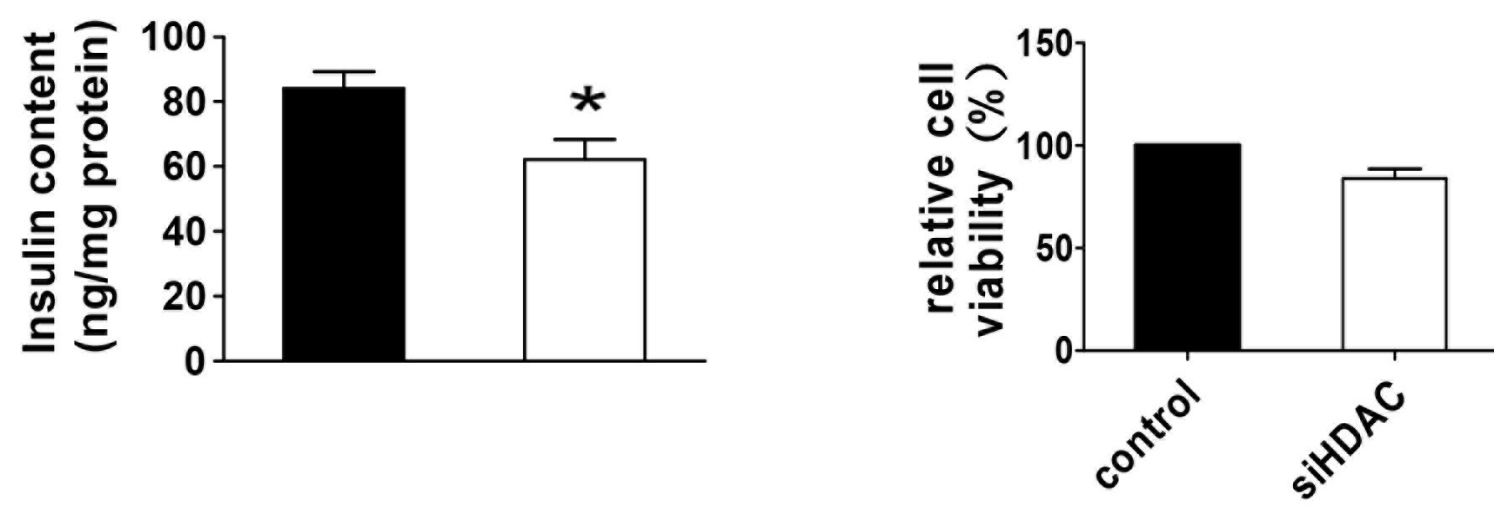

Figure 4: Histopathological analysis of islets of $\boldsymbol{\beta}$-cell-specific HDAC3 KO mice. Pancreatic sections were stained with hematoxylin and eosin from WT and KO mice without STZ treatment A. and B. or 25 days after STZ injection C. and D., scale bar, 100 $\mu \mathrm{m}$.). Islet area relative to total pancreas was evaluated and compared between KO and WT mice of untreated E., or 25 days after STZ treatment F. $n=10$-13 mice per group. Pancreatic insulin content was measure and compared between 8-12-week-old untreated KO and WT mice. $n=4-5$ mice per group G.. The relative viability of Min6 cells transfected with non-targeting sequence siRNA or HDAC3specific siRNA was compared using MTT assay H.. $(* p<0.05, * * p<0.01)$. 
a trend of decreased overall islet area in HDAC3 $\mathrm{KO}$ mice was identified (Figure 4A, 4B and 4E $p=0.07$ ). Meanwhile, HDAC3 KO mice showed significantly decreased pancreatic insulin content, in consistent with the trend of decreased islet $\beta$-cell mass (Figure $4 \mathrm{G}$ ). To further evaluate the potential role of HDAC3 in $\beta$-cell viability, MTT assay was performed to compare the viability of Min-6 cells with HDAC3 silence or control, and no significant difference was identified (Figure 4H). Nevertheless, after STZ treatment, the histological islet $\beta$-cell mass decreased dramatically in HDAC3 KO mice, in consistent with their dramatically increased blood glucose level and diabetes incidence (Figure 4C, 4D and 4F; Figure 3). Taken together, these results strongly suggest that in addition to the potential involvement in islet $\beta$-cell development, HDAC3 also plays important roles in $\beta$-cell insulin secretion and homeostasis.

\section{Direct regulation of SOCS3 by HDAC3 in islet $\beta$-cells contributes to insulin production}

To investigate the potential molecular mechanisms involved in the insulin secretion defect of HDAC3 depleted $\beta$-cells, Min6 cells were transfected with HDAC3 siRNA. The expression of classical insulin relevant genes as well as genes involved in insulin secretory pathways and potentially regulated by HDAC3 was evaluated (Figure 5A). The mRNA level of SOCS3 was significantly increased in both HDAC3 silenced Min6 cells and islets from RIP-Cre ${ }^{+} \mathrm{HDAC}^{\mathrm{f} / \mathrm{fl}}$ mice (Figure $5 \mathrm{~A}$ $\& 5 B)$. To explore the potential regulatory role of HDAC3 on SOCS3, CHIP assay was performed to detect the potential binding of HDAC3 to the promoter of SOCS3. Immunoprecipitation of HDAC3 with anti-HDAC3 was able to robustly pull down the promoter of SOCS3 gene compared with rabbit IgG control antibody (Figure 5C). This result shows the direct binding of HDAC3 to SOCS3 gene, indicating the direct regulatory role of HDAC3 on SOCS3 expression. To further confirm the regulatory role of HDAC3 and the involvement of SOCS3 in HDAC3 deficiency induced $\beta$-cell dysfunction, Min6 cells were co-transfected with siRNAs against HDAC3 and SOCS3. Seventy-two hours after transfection, a flow cytometry analysis was performed to confirm the silence efficiency (Figure 5D). As expected, HDAC3 silence interrupted the transcription of both insulin1 and insulin2. In contrast, SOCS3 silence increased insulin transcription in Min6 cells. Nevertheless, HDAC3 and SOCS3 co-silence reversed the inhibitory effect of HDAC3 silence on insulin transcription, but only insulin 2 mRNA change reach the statistical significant level (Figure 5E). Furthermore, in agreement with the insulin mRNA changes, SOCS3 silence elevate the insulin content, while the co-silence of SOCS3 and HDAC3 rescue the decreased insulin content and secretion induced by HDAC3 silence (Figure 5F \&
$5 \mathrm{G})$.

\section{DISCUSSION}

HDAC3 is essential for embryonic development $[4,5]$. It associates with numerous transcription factors, co-repressors, class II HDACs [7, 16, 17], and plays critical roles in multiple organ development, metabolic homeostasis and function $[8,11]$. In the current study, we made mouse model with conditional deletion of HDAC3 in islet $\beta$-cells to investigate the roles of HDAC3 in islet $\beta$-cell development and function [18, 19]. Mice with $\beta$-cell-specific HDAC3 deletion displayed decreased insulin content, disrupted glucose-stimulated insulin secretion and tolerance, and dramatically enhanced susceptibility to low dose STZ induced diabetes. These data strongly indicate the important role of HDAC3 in islet $\beta$-cell insulin secretion capacity and vulnerability of $\beta$-cells to toxic and inflammatory attack in addition to its potential involvement in $\beta$-cell differentiation. Furthermore, Min6 cells with siRNA mediated HDAC3 silence showed decreased insulin gene transcription, which was mediated, at least partially, through up-regulation of SOCS3. These results not only confirm the critical role of HDAC3 in $\beta$-cell insulin secretion function, but also illuminate the potential molecular mechanisms of HDAC3 mediated islet $\beta$-cell functional regulation. Together, studies from both in vivo and in vitro models indicate that HDAC 3 is essential for proper islet $\beta$-cell differentiation, function and maintenance.

The roles of HDACs in pancreatic developmental regulation remain largely unknown. Class I and class II HDACs are expressed in the embryonic pancreas. The pancreatic expression of HDACs are developmentally regulated, with significantly decreased most class I and II HDAC expression upon differentiation [20], consistent with the regulated HDACs expression during osteogenesis and adipogenesis $[21,22]$. In contrast to other HDACs, HDAC3 expression was dramatically up-regulated in adult pancreas compared to different embryonic stages, suggesting the potential role of HDAC3 in not only pancreatic development, cell differentiation but also their function and maintenance [20].

Treatment of rat embryonic explants with HDAC inhibitors (HDACi) ex vivo promoted the NGN3 proendocrine lineage development, leading to an increased pool of endocrine progenitors. However, different HDACi showed opposing effects on the endocrine $\beta / \delta$ lineage differentiation, in which preferential Class I HDACi VPA/ MS275 induced a dramatic decrease in Pax4 and insulin expression, while the Class I and II HDACi TSA/NaB enhanced Pax4 and insulin expression, suggesting the specific roles of individual HDACs in the development and differentiation of pancreatic $\beta$-cells [20]. Consistent with these results, mice with $\beta$-cell specific HDAC3 deletion in our study showed decreased pancreatic insulin content, 
A

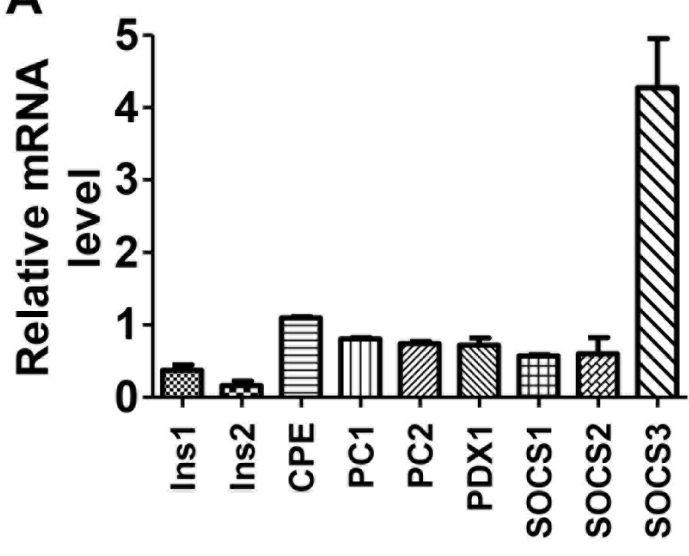

C

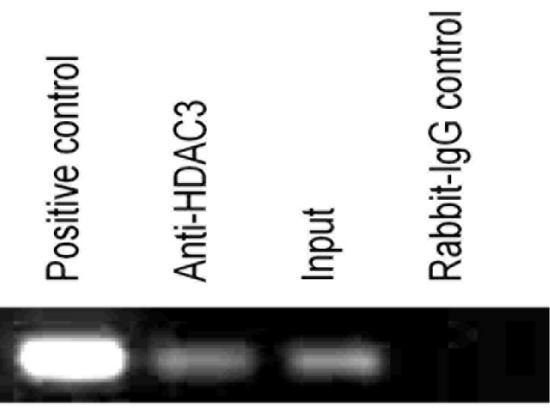

E
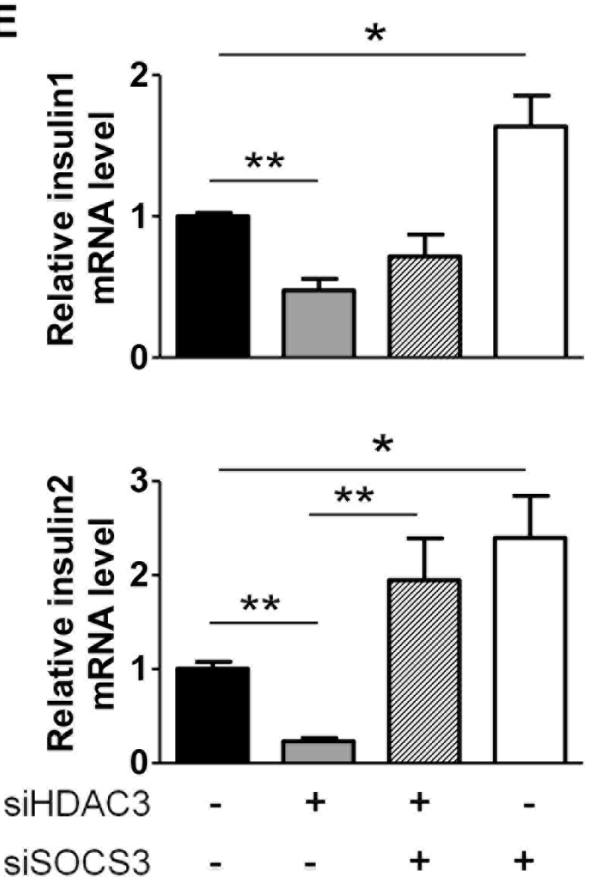

B

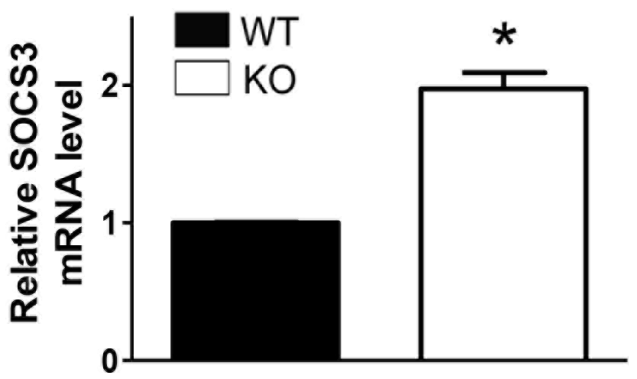

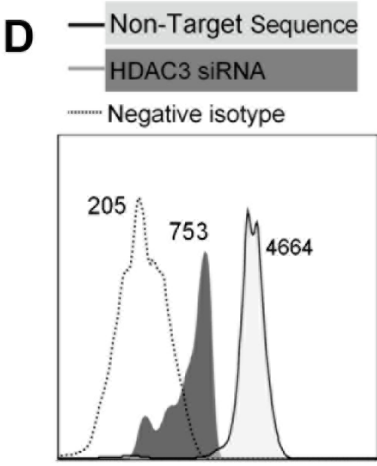

HDAC3-PE

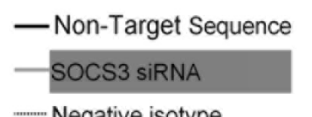

-w-we Negative isotype

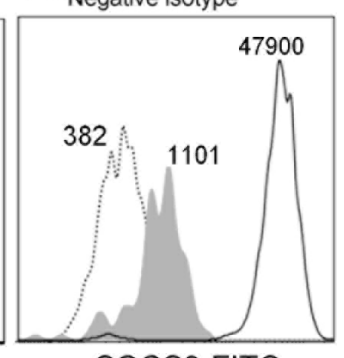

SOCS3-FITC
F

G
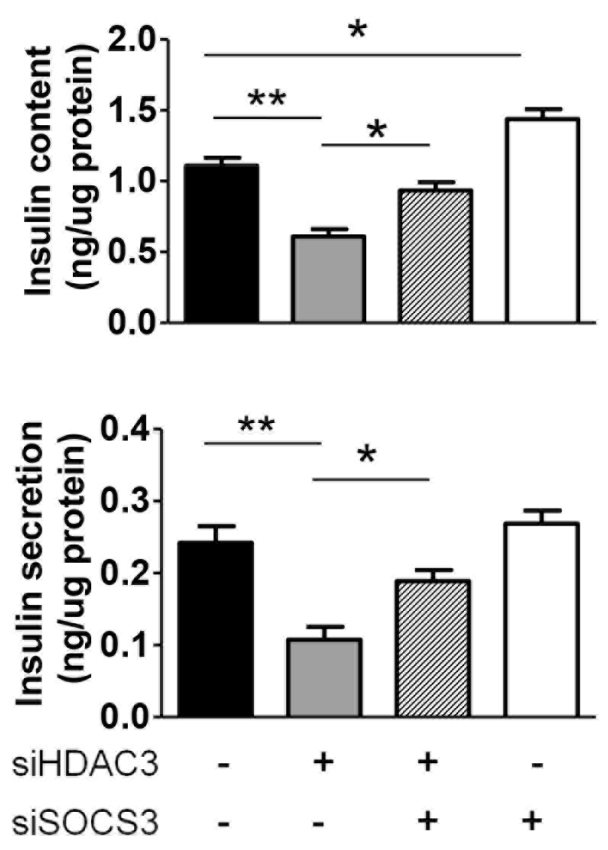

Figure 5: SOCS3 upregulation by HDAC3 depletion in islet $\beta$-cells contributes to insulin production defect. A. Real-time PCR analysis of insulin related gene mRNA changes in HDAC3 silenced Min6 cells. B. Real-time PCR analysis of changed SOCS3 mRNA levels in HDAC3 KO and WT control mice. C. CHIP assays were performed with Min6 cells. Chromatin was immunoprecipitated with antiHDAC3 or rabbit IgG control antibody, and precipitated DNA was used as a template to detect the SOCS3 promoter. Nonimmunoprecipitated sample served as an input control. D. Flow cytometry analysis of Min6 cells with HDAC3 or SOCS3 silencing. E. Real-time PCR analysis of Insulin1 and Insulin2 mRNA levels in different siRNA transfected Min6 cells. Insulin content $\mathbf{F}$. and glucose stimulated insulin secretion F. of Min6 cells with silenced HDAC3, SOCS3 or both. Data are presented as means \pm SD. $\left({ }^{*} p<0.05,{ }^{* *} p<0.01\right)$. 
trend of decreased $\beta$-cell mass, moderate hyperglycemia, which could result, at least partially, from the interrupted $\beta$-cell linage differentiation induced by HDAC3 deletion.

HDAC3 is among the $\beta$-cell expressed HDACs down-regulated by inflammatory cytokine treatment of both primary $\beta$-cells and $\beta$-cell lines $[6,23]$. PanHDACi ITF2357 treatment dose-dependently reversed cytokine induced $\beta$-cell death and the impairment of insulin secretion in INS- 1 cells and primary rat $\beta$-cells, respectively; whereas ITF2357 itself had no effect on primary rat $\beta$-cell insulin secretion and survival, except for high dose of ITF2357 showing a trend of promoting effects on INS-1 apoptosis [6]. Using individual HDAC deleted INS-1 cells or primary rat $\beta$-cells, a more recent study further confirmed that HDAC1 and HDAC3 deletion can partially rescue cytokine-induced $\beta$-cell apoptosis. Nevertheless, the individual HDAC deletion itself had no effects on $\beta$-cell survival [15]. Multiple low dose-streptozotocin (MLD-STZ) induces diabetes through primary $\beta$-cell toxicity plus secondary local inflammation [24]. In the current study, mice with $\beta$-cell-specific HDAC3 deletion displayed significantly enhanced susceptibility to MLD-STZ treatment compared to littermate controls, which could result from either interrupted primary $\beta$-cell differentiation and function, or the increased sensitivity of $\beta$-cells to the toxicity of STZ and inflammatory factors, or both. This result is inconsistent with aforementioned protective role of HDACis in inflammatory cytokine mediated $\beta$-cell apoptosis and functional defects. Comparing these studies with our model system, there are a few possible explanations for the apparent discrepancies: first, Cremediated recombination of HDAC3 in $\beta$-progenitor cells is a permanent event and the phenotypes may result from HDAC3 deletion-mediated $\beta$-cell development, differentiation as well as mature $\beta$-cell function and/or maintenance defects. In contrast, small molecule HDACis have short half-life in serum or culture medium and might only transiently change chromatin structure and gene expression in normally developed $\beta$-cells in relatively short-term assays. Second, HDACs participate in multiprotein transcriptional complexes. Genetic deletion of an HDAC perturbs the complexes in which it would normally be associated, whereas inhibitors are believed to block enzymatic activity without necessarily disrupting the repressive complex. Furthermore, striking difference exist in etiopathogenesis of $\beta$-cell destructions induced by cytokine treatment versus MLD-STZ which include primary $\beta$-cell toxicity from STZ plus secondary local inflammation [24].

SOCS are intracellular proteins that control signals from cytokine receptors and are essential for immune physiology. SOCS proteins are induced by cytokines and act to inhibit cytokine signal transduction. SOCS3 has been reported to protect against cytokine mediated $\beta$-cell destruction by suppressing IFN- $\gamma$ and IL-1 $\beta$ signaling in vitro $[25,26]$. In mice, however, $\beta$-cell specific overexpression of SOCS3 failed to protect against MLDSTZ induced diabetes [27]. Leptin-mediated SOCS3 upregulation transcriptionally inhibits preproinsulin gene expression in $\beta$-cells [28]. In addition to the impact of SOCS3 on $\beta$-cell insulin production, SOCS3 inhibits GH-mediated $\beta$-cell proliferation, and mice with SOCS3 overexpression showed over $30 \% \beta$-cell mass reduction [29]. However, mice with haploinsufficiency of SOCS3 showed enhanced leptin sensitivity and were protected against the development of diet-induced obesity and associated metabolic complications [30]. Furthermore, elevated SOCS3 expression suppressed insulin signaling through inhibiting the phosphorylation of insulin receptor (IR) and insulin receptor substrates (IRS), while promoting the degradation of IRS1 and IRS2 [31-33] These results clearly demonstrate the essential role of SOCS3 in balancing islet $\beta$-cell mass, function and even insulin sensitivity. In the current study, siRNA silencing of HDAC3 dramatically upregulate SOCS3 expression in Min6 cells, in concord with interrupted insulin production, while SOCS3 silencing partially reverse the down regulation of insulin transcription. These results indicate the critical involvement of SOCS3 in HDAC3 deletion induced $\beta$-cell functional defects. Nevertheless, we cannot rule out the possibilities of the involvement of additional genes whose activation or expression is under the control of $\mathrm{HDAC} 3$

HDAC3-containing N-CoR/SMRT complexes bind preferentially to hypoacetylated histones [34, 35]. However, class I HDACs not only remove acetyl groups from histones tails but can also deacetylate transcription factors and other chromatin-associated proteins. Thus, class I HDACs can affect gene transcription at different levels [36]. Recent studies have shown the evidence of HDAC mediated SOCS3 expression regulation: HDACi treatment can promote the SOCS1 and SOCS3 expression in human colorectal cancer and human leukemia through inducing the hyperacetylation of histones associated with the SOCS3 promoters [37, 38], which further suppress Janus kinase/signal transducers and activators of transcription (JAK/STAT) signaling involved in the oncogenesis of different cancers [39]. Thus, these results link SOCS proteins with the anti-tumor functions of HDACis, which further support our findings that SOCS3 is one of the major molecules involved in HDAC3-mediated islet $\beta$-cell differentiation, and function. In addition, our CHIP data suggested the direct regulatory role of HDAC3 on SOCS3 expression. These data combined with previous identified hyper acetylated histone-associated SOCS3 expression regulation indicate the existence of complex multi-pathways in HDAC mediated SOCS protein regulation including at least the conventional Histone deacetylation-mediated and the direct regulation pathway possibly recruited by SOCS3 regulatory transcription factors. The detailed molecular mechanisms of SOCS3- 
dependent and -independent pathways of HDAC3 related islet $\beta$-cell development, differentiation and functional regulation will be further investigated in future studies.

In summary, using the islet $\beta$-cell specific HDAC3 deletion mouse model combined with HDAC3 silenced Min6 cell line, our results indicate the critical role of HDAC3 in islet $\beta$-cell differentiation, function and maintenance, and the role of SOCS3 in HDAC3 deletion induced $\beta$-cell dysfunction. These results not only provide further insight into the epigenetic mechanisms of $\beta$-cell development and functional regulation, but also emphasize the importance of understanding the role and mechanisms of individual HDACs in $\beta$-cell regulation, for future HDAC related clinical applications with increased specificity and minimized toxicity in the treatment and prevention of diabetes mellitus and other diseases.

\section{MATERIALS AND METHODS}

\section{Islet isolation, dispersion, immunofluorescence staining, and pancreas histological and immunofluorescence analysis}

Mouse islets were isolated by type $\mathrm{V}$ collagenase (Sigma Chemicals, St Louis, MO, USA) digestion of pancreas. Isolated islets were exposed to $0.25 \%$ trypsinEDTA at $37^{\circ} \mathrm{C}$ for $10 \mathrm{~min}$, followed by gently pipetting for 1-2 $\mathrm{min}$ to dissociate into single cells. Dispersed islets cells were fixed in 4\% (vol./vol.) paraformaldehyde for $10 \mathrm{~min}$, permeabilized with $0.25 \%$ (vol./vol.) Triton $\mathrm{X}-100$ for $10 \mathrm{~min}$ and blocked overnight in $1 \%$ (wt/vol.) BSA at $4^{\circ} \mathrm{C}$. Cells were then labeled overnight at $4^{\circ} \mathrm{C}$ with polyclonal anti-HDAC3 (Abcam, Cambridge, MA, USA) and monoclonal anti-insulin (Linco, Research, Inc., St Louis, MO, USA) antibodies. Cells were then stained at room temperature in dark for $60 \mathrm{~min}$ with PEconjugated goat anti-rabbit, FITC-conjugated goat antimouse secondary antibodies (eBioscience, San Diego, CA, USA) respectively. Cells were washed and mounted on glass slides which were viewed on a microscope (Leica Microsystems, Wetzlar, Germany).

Pancreases obtained from 8- to 12-week-old mice were used for morphometry and immunostaining. For histological analysis, pancreases were fixed in $10 \%$ formalin, paraffin-embedded, and sectioned in $6 \mu \mathrm{m}$ (each section spaced at least $50 \mu \mathrm{m}$ apart), 4 sections of each animal were used. Pancreas sections were stained with hematoxylin and eosin for histological study. To measure islet area, sections were photographed at low magnification. Images were imported to a software (ImageJ, NIH, Bethesda, MA, USA), and total pancreas and islet areas were selected. Pixel numbers in selected areas were used to calculate the relative islet area to total pancreas.

\section{Glucose tolerance test}

Mice were subjected to glucose tolerance test at 8-10 weeks of age. Mice were fasted overnight (16 hours) and intraperitoneally injected with $0.9 \% \mathrm{NaCl}$ solution containing D-glucose at the dose of 1 or $2 \mathrm{~g} / \mathrm{kg}$ body weight. Blood samples for glucose measurements were taken at $0,5,10,15,30,60$ and 120 minutes after the glucose load. Serum was also collected at 0, 5, 30 minutes after glucose load, insulin concentration was detected using mouse insulin ELISA Kit (Crystal Chem Inc., Chicago, Illinois, USA)

\section{Multiple low-dose streptozotocin (MLD-STZ) treatment}

Mice were intraperitoneal injected with STZ (Sigma Chemicals, St Louis, MO, USA) of $40 \mathrm{mg} / \mathrm{kg}$ body weight for five consecutive days. Blood glucose levels were measured twice a week using a glucometer (FreeStyle, Abbott, Alameda, CA, USA). Mice were considered diabetic when blood glucose levels were $>200 \mathrm{mg} / \mathrm{dl}$ in two consecutive measurements. Mice were killed on day 25 of the study; pancreases and serum were collected and used for further evaluation.

\section{Cell culture and RNA interference}

Min-6 cells were cultured in $25 \mathrm{mmol} / 1$ glucose Dulbecco's modified Eagle's medium (DMEM), supplemented with $15 \%$ fetal bovine serum, $50 \mu \mathrm{M}$ $\beta$-mercaptoethanol, 100 units $/ \mathrm{ml}$ penicillin, $100 \mu \mathrm{g} / \mathrm{ml}$ streptomycin, and $2 \mathrm{mmol} / 1 \mathrm{l}$-glutamine at $37^{\circ} \mathrm{C}, 5 \% \mathrm{CO}_{2}$. Cells were trypsinized, counted, and plated in culture medium without antibiotics at a density of $\sim 40-50 \%$, 24 hours before transfection. The on-target HDAC3 and Suppressors of cytokine signaling 3 (SOCS3) siRNAs (Thermo Scientific, Lafayette, CO, USA) as well as nontargeting sequence siRNA were transfected at a working concentration of $50 \mathrm{nM}$ using Lipofectamine RNAiMax (Invitrogen, Carlsbad, CA, USA) according to the manufacturer's instruction. Transfected cells were cultured for $72 \mathrm{hrs}$ for glucose-stimulated insulin secretion, flowcytometry analysis and Real-time PCR.

\section{RNA isolation and quantitative real-time PCR}

Total RNAs were isolated using Qiazol (Qiagen, Valencia, CA, USA) and subjected to reverse transcription using High Capacity cDNA Reverse Transcription Kit ((Applied Biosystems, Foster City, CA, USA). Realtime PCR was performed in triplicates using FastStart Universal SYBR Green Master (Roche Diagnostics Corp., Indiana, USA) on ABI 7900 HT Real-Time PCR system 
(Applied Biosystems, Foster City, CA, USA). Data were normalized to mouse $\beta$-actin mRNA.

\section{Chromatin immunoprecipitation (CHIP) assay}

CHIP assays were performed using an EZ-CHIP kit (Millipore, Billerica, MA, USA), following the manufacturer's instructions. CHIP-grade anti-HDAC3 (Abcam, Cambridge, MA, USA), rabbit IgG negative control antibody (eBioscience, San Diego, CA, USA) and anti-RNA polymerase II (Millipore, Billerica, MA, USA) were used to immunoprecipitate the protein-DNA complexes. Purified immunoprecipitated DNA was analyzed by PCR with primers for SOCS3 promoter (Table S1).

\section{Glucose-stimulated insulin secretion and insulin content test}

The cultured cells were washed and pre-incubated for $60 \mathrm{~min}$ in Krebs-Ringer bicarbonate buffer (129.4 $\mathrm{mmol} / 1 \mathrm{NaCl}, 5.2 \mathrm{mmol} / 1 \mathrm{KCl}, 1.3 \mathrm{mmol} / 1 \mathrm{KH}_{2} \mathrm{PO}_{4}, 2.7$ $\mathrm{mmol} / 1 \mathrm{CaCl}_{2}, 1.3 \mathrm{mmol} / 1 \mathrm{MgSO}_{4}, 24.8 \mathrm{mmol} / 1 \mathrm{NaHCO}_{3}$, $10 \mathrm{mmol} / 1 \mathrm{HEPES}, 0.1 \% \mathrm{BSA}$ [pH 7.4]) with $2.8 \mathrm{mmol} / \mathrm{L}$ glucose. Upon completion of the incubation, the buffer was removed completely and replaced with fresh KRB containing $20 \mathrm{mmol} / \mathrm{L}$ glucose for an additional $45 \mathrm{~min}$. The supernatant was collected for measuring insulin secretion.

The total insulin in the cells as well as the whole pancreas was extracted by acid ethanol $(0.18 \mathrm{M} \mathrm{HCl}$ in $95 \%$ ethanol) treatment overnight. Insulin concentration was measured using mouse insulin ELISA Kit (Crystal Chem Inc., Chicago, Illinois, USA). Insulin secretion and content were normalized to total protein.

\section{Flow cytometry (FACS)}

Min-6 cells were trypsinized, washed twice with FACS staining buffer (PBS, 3\% FBS), and then incubated with Fc block (clone 2.4G2). Trypsinized cells were first fixed and permeabilized by Foxp3 Fixation Buffer and Permeabilization Buffer (eBioscience), and then incubated with Fc blocker (clone 24G2). Treated cells were then stained with related antibodies: anti-HDAC3 (ab7030, Abcam), anti-SOCS3 (ab16030, Abcam), or rabbit IgG as isotype control, followed by incubation with FITC or PEconjugated anti-rabbit IgG (eBioscience, 1:100 dilution). Data was harvested by FACSAriaII Flow Cytometer (BD Biosciences) and analyzed using FlowJo software (FlowJo, LLC).

\section{Statistical analysis}

Statistical analysis was performed with Prism 5.0 (GraphPad Software, San Diego, CA). A two-tailed Student $t$ test was used to test the differences between two groups. ANOVA was used to compare the means of multiple groups. Data are presented as mean \pm standard deviation (SD). Gehan-Breslow-Wilcoxon test was used for the analysis of the incidence of diabetes. Differences were considered significant with a $p$ value $<0.05$.

\section{ACKNOWLEDGMENTS}

We thank Dr. Scott W. Hiebert for HDAC $3^{\mathrm{f} / \mathrm{f}}$ mice, and all members of our laboratory for their advice and encouragement.

\section{CONFLICTS OF INTREST}

The authors declare that there is no conflict of interest associated with this manuscript.

\section{GRANT SUPPORT}

This study was supported in part by Juvenile Diabetes Research Foundation International grants (12007-039, 5-2006-403), and Henry Ford Immunology Program star-up Funds (T71016 and T71017).

\section{REFERENCES}

1. Grunstein M. Histone acetylation in chromatin structure and transcription. Nature. 1997; 389(6649):349-352.

2. Choudhary C, Kumar C, Gnad F, Nielsen ML, Rehman M, Walther TC, Olsen JV and Mann M. Lysine acetylation targets protein complexes and co-regulates major cellular functions. Science. 2009; 325(5942):834-840.

3. de Ruijter AJ, van Gennip AH, Caron HN, Kemp S and van Kuilenburg AB. Histone deacetylases (HDACs): characterization of the classical HDAC family. Biochem J. 2003; 370(Pt 3):737-749.

4. Haberland M, Montgomery RL and Olson EN. The many roles of histone deacetylases in development and physiology: implications for disease and therapy. Nat Rev Genet. 2009; 10(1):32-42.

5. Bhaskara S, Chyla BJ, Amann JM, Knutson SK, Cortez D, Sun ZW and Hiebert SW. Deletion of histone deacetylase 3 reveals critical roles in $\mathrm{S}$ phase progression and DNA damage control. Mol Cell. 2008; 30(1):61-72.

6. Lundh M, Christensen DP, Rasmussen DN, Mascagni P, Dinarello CA, Billestrup N, Grunnet LG and Mandrup-Poulsen T. Lysine deacetylases are produced in pancreatic beta cells and are differentially regulated by proinflammatory cytokines. Diabetologia. 2010; 
53(12):2569-2578.

7. Li J, Wang J, Nawaz Z, Liu JM, Qin J and Wong J. Both corepressor proteins SMRT and N-CoR exist in large protein complexes containing HDAC3. EMBO J. 2000; 19(16):4342-4350.

8. Montgomery RL, Potthoff MJ, Haberland M, Qi X, Matsuzaki S, Humphries KM, Richardson JA, BasselDuby R and Olson EN. Maintenance of cardiac energy metabolism by histone deacetylase 3 in mice. J Clin Invest. 2008; 118(11):3588-3597.

9. Sun Z, Miller RA, Patel RT, Chen J, Dhir R, Wang H, Zhang D, Graham MJ, Unterman TG, Shulman GI, Sztalryd C, Bennett MJ, Ahima RS, Birnbaum MJ and Lazar MA. Hepatic Hdac3 promotes gluconeogenesis by repressing lipid synthesis and sequestration. Nat Med. 2012; 18(6):934-942.

10. Knutson SK, Chyla BJ, Amann JM, Bhaskara S, Huppert SS and Hiebert SW. Liver-specific deletion of histone deacetylase 3 disrupts metabolic transcriptional networks. EMBO J. 2008; 27(7):1017-1028.

11. Razidlo DF, Whitney TJ, Casper ME, McGee-Lawrence ME, Stensgard BA, Li X, Secreto FJ, Knutson SK, Hiebert SW and Westendorf JJ. Histone deacetylase 3 depletion in osteo/chondroprogenitor cells decreases bone density and increases marrow fat. PLoS One. 2010; 5(7):e11492.

12. Lewis EC, Blaabjerg L, Storling J, Ronn SG, Mascagni P, Dinarello CA and Mandrup-Poulsen T. The oral histone deacetylase inhibitor ITF2357 reduces cytokines and protects islet beta cells in vivo and in vitro. Mol Med. 2011; 17(5-6):369-377.

13. Chou DH, Holson EB, Wagner FF, Tang AJ, Maglathlin RL, Lewis TA, Schreiber SL and Wagner BK. Inhibition of histone deacetylase 3 protects beta cells from cytokineinduced apoptosis. Chem Biol. 2012; 19(6):669-673.

14. Patel T, Patel V, Singh R and Jayaraman S. Chromatin remodeling resets the immune system to protect against autoimmune diabetes in mice. Immunol Cell Biol. 2011; 89(5):640-649.

15. Lundh M, Christensen DP, Damgaard Nielsen M, Richardson SJ, Dahllof MS, Skovgaard T, Berthelsen J, Dinarello CA, Stevenazzi A, Mascagni P, Grunnet LG, Morgan NG and Mandrup-Poulsen T. Histone deacetylases 1 and 3 but not 2 mediate cytokine-induced beta cell apoptosis in INS-1 cells and dispersed primary islets from rats and are differentially regulated in the islets of type 1 diabetic children. Diabetologia. 2012; 55(9):2421-2431.

16. Fischle W, Dequiedt F, Hendzel MJ, Guenther MG, Lazar MA, Voelter W and Verdin E. Enzymatic activity associated with class II HDACs is dependent on a multiprotein complex containing HDAC3 and SMRT/N-CoR. Mol Cell. 2002; 9(1):45-57.

17. Guenther MG, Yu J, Kao GD, Yen TJ and Lazar MA. Assembly of the SMRT-histone deacetylase 3 repression complex requires the TCP-1 ring complex. Genes Dev.
2002; 16(24):3130-3135.

18. Slack JM. Developmental biology of the pancreas. Development. 1995; 121(6):1569-1580.

19. Gannon M, Shiota C, Postic C, Wright CV and Magnuson M. Analysis of the Cre-mediated recombination driven by rat insulin promoter in embryonic and adult mouse pancreas. Genesis. 2000; 26(2):139-142.

20. Haumaitre $\mathrm{C}$, Lenoir $\mathrm{O}$ and Scharfmann R. Histone deacetylase inhibitors modify pancreatic cell fate determination and amplify endocrine progenitors. Mol Cell Biol. 2008; 28(20):6373-6383.

21. Lee HW, Suh JH, Kim AY, Lee YS, Park SY and Kim JB. Histone deacetylase 1-mediated histone modification regulates osteoblast differentiation. Mol Endocrinol. 2006; 20(10):2432-2443.

22. Yoo EJ, Chung JJ, Choe SS, Kim KH and Kim JB. Downregulation of histone deacetylases stimulates adipocyte differentiation. J Biol Chem. 2006; 281(10):6608-6615.

23. Ortis F, Naamane N, Flamez D, Ladriere L, Moore F, Cunha DA, Colli ML, Thykjaer T, Thorsen K, Orntoft TF and Eizirik DL. Cytokines interleukin-1beta and tumor necrosis factor-alpha regulate different transcriptional and alternative splicing networks in primary beta-cells. Diabetes. 2010; 59(2):358-374.

24. Wilson GL and Leiter EH. Streptozotocin interactions with pancreatic beta cells and the induction of insulin-dependent diabetes. Current topics in microbiology and immunology. 1990; 156:27-54.

25. Karlsen AE, Heding PE, Frobose H, Ronn SG, Kruhoffer M, Orntoft TF, Darville M, Eizirik DL, Pociot F, Nerup J, Mandrup-Poulsen T and Billestrup N. Suppressor of cytokine signalling (SOCS)-3 protects beta cells against IL-1beta-mediated toxicity through inhibition of multiple nuclear factor-kappaB-regulated proapoptotic pathways. Diabetologia. 2004; 47(11):1998-2011.

26. Karlsen AE, Ronn SG, Lindberg K, Johannesen J, Galsgaard ED, Pociot F, Nielsen JH, Mandrup-Poulsen T, Nerup J and Billestrup N. Suppressor of cytokine signaling 3 (SOCS-3) protects beta -cells against interleukin-1beta and interferon-gamma -mediated toxicity. Proc Natl Acad Sci U S A. 2001; 98(21):12191-12196.

27. Borjesson A, Ronn SG, Karlsen AE, Billestrup N and Sandler S. beta-cell specific overexpression of suppressor of cytokine signalling-3 does not protect against multiple low dose streptozotocin induced type 1 diabetes in mice. Immunol Lett. 2011; 136(1):74-79.

28. Laubner K, Kieffer TJ, Lam NT, Niu X, Jakob F and Seufert J. Inhibition of preproinsulin gene expression by leptin induction of suppressor of cytokine signaling 3 in pancreatic beta-cells. Diabetes. 2005; 54(12):3410-3417.

29. Lindberg K, Ronn SG, Tornehave D, Richter H, Hansen JA, Romer J, Jackerott M and Billestrup N. Regulation of pancreatic beta-cell mass and proliferation by SOCS-3. J Mol Endocrinol. 2005; 35(2):231-243. 
30. Howard JK, Cave BJ, Oksanen LJ, Tzameli I, Bjorbaek C and Flier JS. Enhanced leptin sensitivity and attenuation of diet-induced obesity in mice with haploinsufficiency of Socs3. Nat Med. 2004; 10(7):734-738.

31. Emanuelli B, Glondu M, Filloux C, Peraldi P and Van Obberghen E. The potential role of SOCS-3 in the interleukin-1beta-induced desensitization of insulin signaling in pancreatic beta-cells. Diabetes. 2004; 53 Suppl 3:S97-S103.

32. Rui L, Yuan M, Frantz D, Shoelson S and White MF. SOCS-1 and SOCS-3 block insulin signaling by ubiquitinmediated degradation of IRS1 and IRS2. J Biol Chem. 2002; 277(44):42394-42398.

33. Ueki K, Kondo T and Kahn CR. Suppressor of cytokine signaling 1 (SOCS-1) and SOCS-3 cause insulin resistance through inhibition of tyrosine phosphorylation of insulin receptor substrate proteins by discrete mechanisms. Mol Cell Biol. 2004; 24(12):5434-5446.

34. Vermeulen M, Walter W, Le Guezennec X, Kim J, Edayathumangalam RS, Lasonder E, Luger K, Roeder RG, Logie C, Berger SL and Stunnenberg HG. A feed-forward repression mechanism anchors the Sin3/histone deacetylase and N-CoR/SMRT corepressors on chromatin. Mol Cell Biol. 2006; 26(14):5226-5236.
35. Yoon HG, Choi Y, Cole PA and Wong J. Reading and function of a histone code involved in targeting corepressor complexes for repression. Mol Cell Biol. 2005; 25(1):324335.

36. Moser MA, Hagelkruys A and Seiser C. Transcription and beyond: the role of mammalian class I lysine deacetylases. Chromosoma. 2014; 123(1-2):67-78.

37. Xiong $\mathrm{H}, \mathrm{Du} \mathrm{W}$, Zhang YJ, Hong J, Su WY, Tang JT, Wang YC, Lu R and Fang JY. Trichostatin A, a histone deacetylase inhibitor, suppresses JAK2/STAT3 signaling via inducing the promoter-associated histone acetylation of SOCS1 and SOCS3 in human colorectal cancer cells. Mol Carcinog. 2012; 51(2):174-184.

38. Gao SM, Chen CQ, Wang LY, Hong LL, Wu JB, Dong PH and Yu FJ. Histone deacetylases inhibitor sodium butyrate inhibits JAK2/STAT signaling through upregulation of SOCS1 and SOCS3 mediated by HDAC8 inhibition in myeloproliferative neoplasms. Experimental hematology. 2013; 41(3):261-270 e264.

39. Quintas-Cardama A and Verstovsek S. Molecular pathways: Jak/STAT pathway: mutations, inhibitors, and resistance. Clinical cancer research. 2013; 19(8):1933-1940. 